\title{
Human research ethics in biomedical journals
}

\author{
Keith Ong \\ Asian JO Chief and Managing Editor
}

Human research involves research conducted on human subjects, their personal data, or tissue. This includes clinical trials involving testing and treatment, surveys which involve personal data, interviews, or observation, and blood or tissue specimens.

Looking through the history of research in medicine and science, we find that some experiments compromised the well-being of human research subjects. In World War II concentration camps, experiments were conducted without the consent of the subjects and involved extreme pain and suffering, many times ending in major disability and death of the research subjects. The Nuremberg Code and the related Declaration of Helsinki arose from the need for guidelines to protect the well-being of research subjects.

The World Medical Association (WMA) Declaration of Helsinki on Ethical Principles for Medical Research Involving Human Subjects was initially adopted by the 18th WMA General Assembly in Helsinki, Finland in June 1964. ${ }^{1}$ It outlines the guidelines for physicians engaged in clinical research focusing on the responsibilities of researchers for the protection of research subjects.

The role of Human Research Ethics Committees (HREC) mainly involves protecting the welfare of human research subjects. This concern also extends to animals, included in item 21 of the Declaration of Helsinki: "Medical research involving human subjects must conform to generally accepted scientific principles, be based on a thorough knowledge of the scientific literature, other relevant sources of information, and adequate laboratory and, as appropriate, animal experimentation. The welfare of animals used for research must be respected."1 The same principles apply to animals as they do to human subjects, that is, the benefits should outweigh the harm.

Over the years, the Declaration of Helsinki has been further elaborated; it was last updated by the 64th WMA General Assembly in Fortaleza, Brazil in October 2013. While becoming more comprehensive, it has also become more difficult for researchers to understand. It may basically be summarized as follows: "To avoid or minimize harm to research subjects; where there is potential harm, it should

Correspondence: Dr. Keith Ong, MBBS, FRACO, FRACS, MMed, PGCert HE, 2 Railway Avenue, Eastwood, NSW 2122, Australia.

E-mail: keithong@asianjo.com 
be made clear to the research subjects and the benefits of the research should outweigh the potential harm."

When there is potential harm, a committee is then needed to decide if the benefits outweigh risks and harm to the subjects so that a comprehensive range of viewpoints has been evaluated, which is the reason why HREC are comprised of members from different vocations. The benefits of research include gains in knowledge, insight, and understanding to improve society and individual well-being as well as gains in expertise for researchers. Direct benefits to research participants include potential improvement in disease due to new treatments. The role of HREC is to protect potential participants involved in research. In doing so, they must take into account the potential risks and benefits for the community in which the research will be carried out. The ultimate goal is to promote high ethical standards in biomedical research.

Harm can be classified into physical, psychological, social, economic, and legal aspects. Discomfort, which can be physical (having blood pressure measured) or psychological (anxiety when being interviewed), is classified as less serious than harm. Less serious again is inconvenience, which may include the time taken to fill out a form or participate in research.

As guidelines become comprehensive, they have also become more complex to navigate. Asian JO would like to simplify and clarify these aspects, as follows:

1. All prospective studies require HREC or Institutional Review Board (IRB) approval; approval should identify the governing body issuing approval (or waiver of) and appropriate document numbers.

2. Retrospective studies do not need to apply for HREC approval if there is no contact (such as interviews) with the patients and/or when a researcher who is not part of the patients' treatment team is provided with de-identified data, ensuring there is no breach of privacy. Retrospective studies may be low/negligible risk (LNR). Low risk research is defined as research in which the only foreseeable risk is one discomfort. Negligible risk research is defined as research in which there is no foreseeable risk of harm or discomfort; and any foreseeable risk is no more than inconvenience. In LNR, if a researcher who is not part of the treatment team has to access a patient's medical records or contact a patient for clarification of medical details, there may be breach of privacy issues. In such instances, consent has to be given by the subject (patient) or guardian for release of information, otherwise there is a need to seek waiver of consent from HREC. Hence, the authors will make a statement that there was no breach of privacy issues or consent has been obtained or a waiver of consent has been approved by HREC. The authors will also make a statement that the study was retrospective in nature, did not alter the routine management of patients, and would not 
alter the outcome of their procedures, which have already been performed prior to the study being conducted.

3. Manuscripts that include an individual patient's clinical information and/or images that may identify the individual, such as case reports and case series, require consent for publication from the subject. The authors are asked to keep this signed consent form in their records. Asian JO asks that authors complete a form declaring that informed consent has been obtained from study subjects.

4. Systematic review papers that review sources already published in journals or information available in the public domain do not need HREC application. We hope that this editorial clarifies guidelines, interpretation and reasons regarding human research ethics requirements for Asian JO, which are also available online in our Ethics and Malpractice Policy. We would also like to inform prospective authors and other interested parties that our Author Guidelines have been updated and come into effect starting May 1, 2021.

Dr. Keith Ong

Chief and Managing Editor

Asian Journal of Ophthalmology

\section{References}

1. World Medical Association. Declaration of Helsinki. Available from: https://www.wma.net/policies-post/ wma-declaration-of-helsinki-ethical-principles-for-medical-research-involving-human-subjects/ 